\title{
Chemical Mapping of Unstained DNA Origami Using STEM/EDS and Graphene Supports
}

\author{
Todd H. Brintlinger, ${ }^{1}$ Susan Buckhout-White, ${ }^{2 *}$ Nabil D. Bassim, ${ }^{1+}$ Divita Ma- \\ thur, ${ }^{3}$ Anirban Samanta, ${ }^{3 \#}$ Jeremy T. Robinson, ${ }^{4}$ Juan-Carlos Idrobo, ${ }^{5}$ Rhonda M. \\ Stroud, ${ }^{1}$ Ellen R. Goldman, ${ }^{2}$ and Mario G. Ancona ${ }^{4 \$}$
}

\begin{abstract}
${ }^{1}$ Materials Sci. \& Tech. Division, Naval Research Laboratory, Washington, DC, 20375, USA; ${ }^{2}$ Center for Biomolecular Sci. \& Eng., Naval Research Laboratory, Washington, DC, 20375, USA; ${ }^{3}$ Dept. Biology, George Mason University, Fairfax, VA, 22030, USA; ${ }^{4}$ Electronics Sci. \& Tech. Division, Naval Research Laboratory, Washington, DC, 20375, USA; ${ }^{5}$ Center for Nanophase Materials Science, Oak Ridge Nat'l Lab., Oak Ridge, TN, 37831, USA; * Current address: Gryphon Schafer, Inc., Arlington, VA, 22203, USA; ${ }^{+}$Current address: Dept. of Materials Science \& Engineering, McMaster Univ., Hamilton, ON, L8S 4L7, Canada; \# Current address: Dept. of Chemistry, Ramakrishna Mission Vidyamandira, West Bengal, India \$Corresponding Author: ancona@estd.nrl.navy.mil
\end{abstract}

\section{S1. Atomic Force Microscopy}

The standard DNA origami in TAE with $\mathrm{MgCl}_{2}$ as well as the origami in TAE with $\mathrm{BaCl}_{2}$ were both imaged to ascertain any intrinsic morphology differences. The sample formed in TAE with $\mathrm{MgCl}_{2}$ shows the expected structure with the single central opening and three small holes at each apex (See Figure 1 in main text). The $\mathrm{MgCl}_{2}$ origami are generally

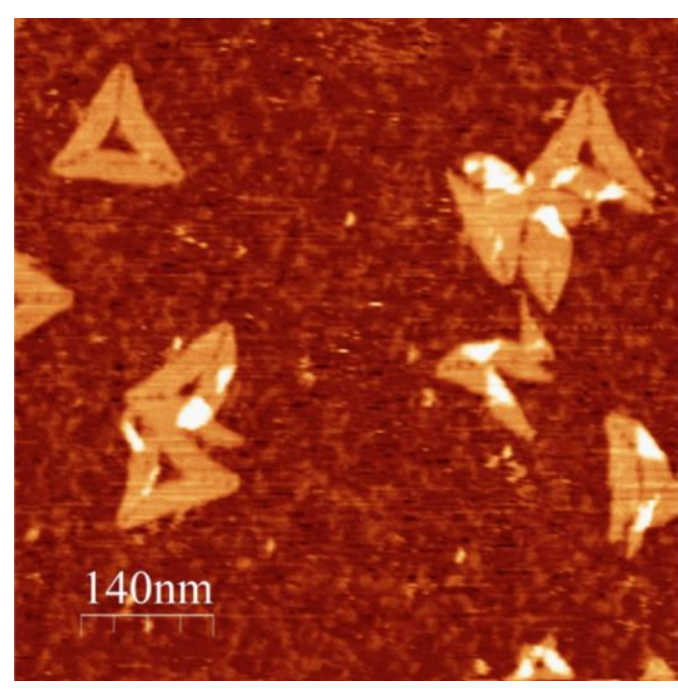

Figure S1. AFM image of the DNA origami triangles made for this paper in $\mathrm{BaCl}_{2}$. 
seen to flat with mostly individual triangles plus a few dimers present. In contrast the origami triangle formed in $\mathrm{BaCl}_{2}$ (see Figure $\mathrm{S} 1$ ) shows much greater adhesion with the mica surface as well as with itself resulting in clustered formations and structures that have folded upon themselves. Few structures are seen in isolation with dimers or larger groupings more common.

\section{S2. Graphene Supports}

The process used to fabricate the graphene supports is depicted in Fig. S2. Also included in the figure are optical micrographs of these supports when ready for use. Fig. S3 contains a TEM image of our 'best practices' graphene prior to use (i.e., with no DNA) and showing the pristine graphene areas as well as carbonaceous residues in between. Fig. S4 shows for comparison a TEM image of a commerical graphene TEM support (from Graphenea [28]), including evidence of multilayers.

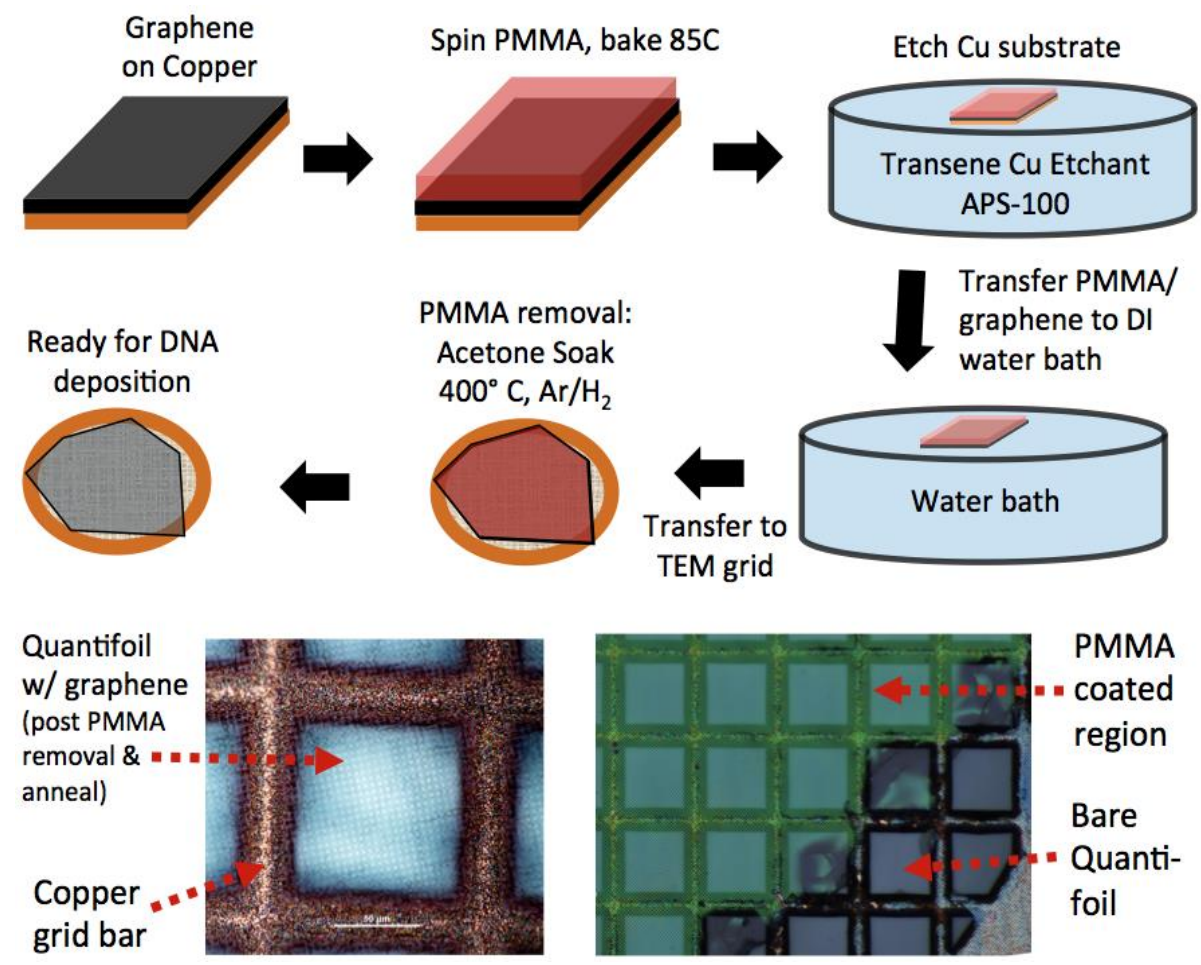

Figure S2. Schematic of the process used to make the graphene supports, and including optical micrographs of their form when ready for use. 


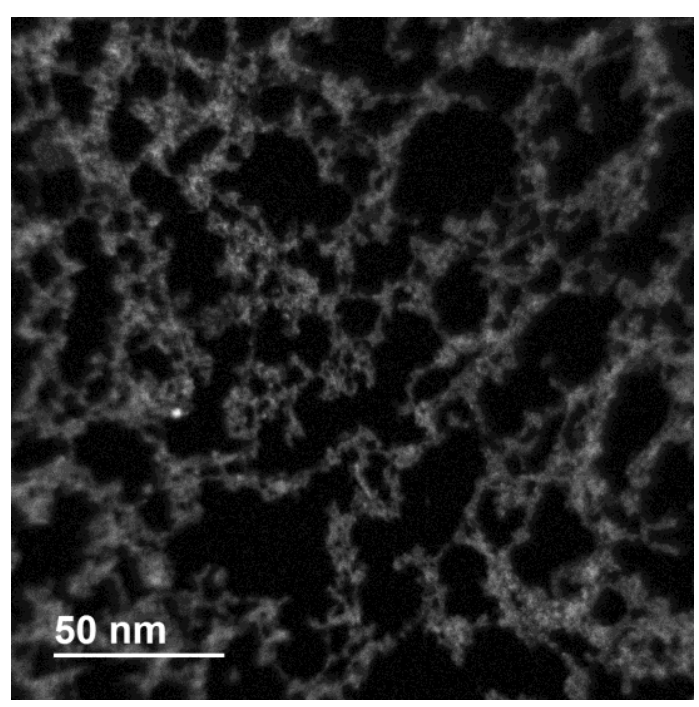

Figure S3. STEM-HAADF image of our 'best practices' graphene with no DNA present.

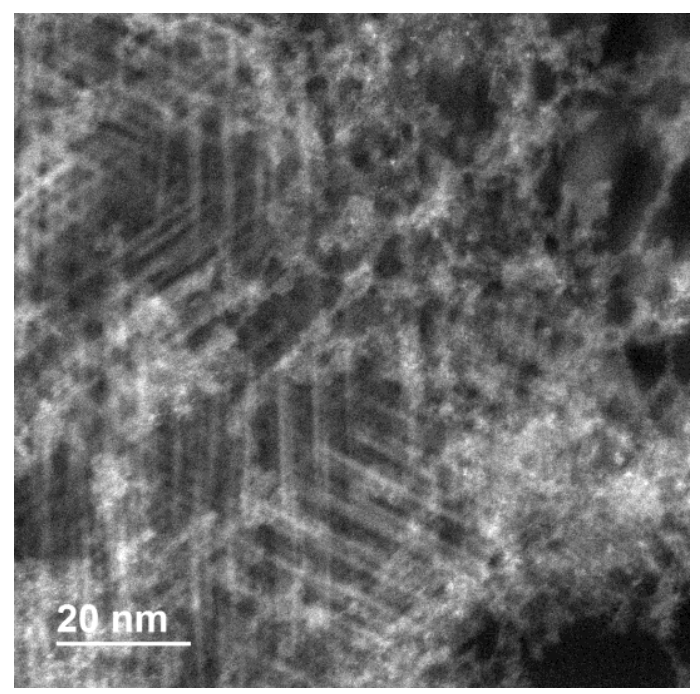

Figure S4. STEM-HAADF image of a commercial graphene TEM support showing evidence of multiple graphene layers in moiré pattern.

Although the carbonaceous residue on our 'best practices' graphene appears not to have any (major) effect on our ability to obtain HAADF/MAADF images or EDS maps of the DNA nanostructures, it could still have role in TEM studies of DNA structures, in particular by affecting the adhesion of the DNA to these supports. To investigate how the carbon contamination affects adhesion, ideally one would want to make a graphene support absent contamination and compare it to one with contamination. Lacking graphene supports without contamination [23], we did two indirect experiments. In the first we compared results of 'best practices' graphene with graphene which had been grown two weeks prior to its transfer/anneal. The result for the latter appears in Fig. S5 and we find that the level of carbon contamination is not significantly increased (compare Fig. 2A). This suggests that the amount of carbon adsorbed during storage in a container in a cleanroom is actually minimal and that separate graphene growth runs for each DNA nanostructure are probably not necessary. In a second experiment we took a graphene sub- 
strate and immediately prior to the TEM step exposed it to a series of plasma treatments intended to remove the overlying carbon contamination. Experimentally we found little difference in TEM images between the untreated and plasma-treated samples (Fig. S6). Finally, in Fig. S7 we show a graphene 'sandwich' sample that had been annealed at $400^{\circ} \mathrm{C}$. Significant degradation is apparent.

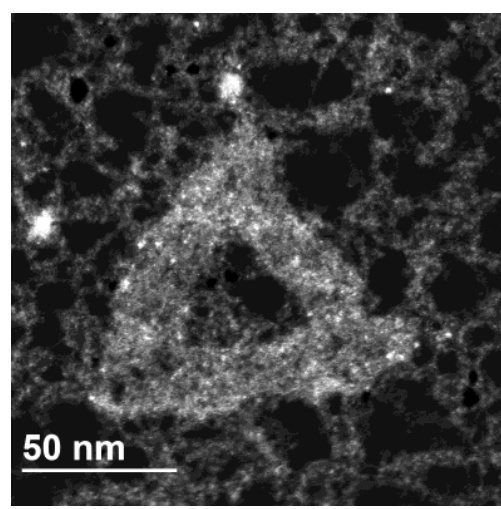

Figure S5. STEM-HAADF image of DNA nanostructure on plasma treated graphene.

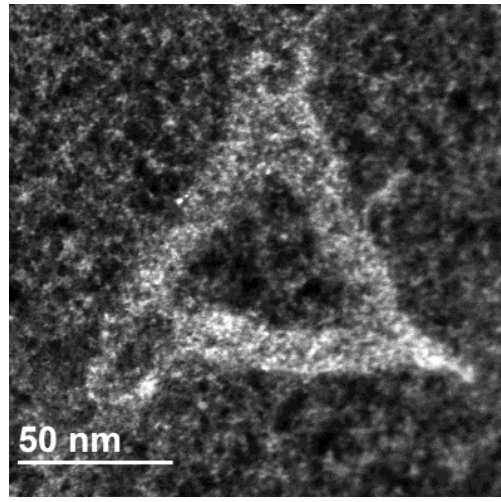

Figure S6. STEM-HAADF image of DNA nanostructure on graphene TEM support in which graphene growth occured two weeks before transfer.

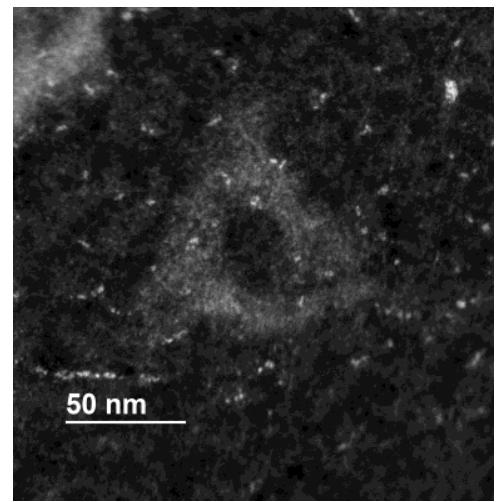

Figure S7: STEM-HAADF image of DNA triangle in a graphene 'sandwich' annealed at $400^{\circ} \mathrm{C}$.

\section{EDS Spectra and Analyses}

For quantitative atomic percent analysis, we perform background subtraction, Bayesian deconvolution, and a Cliff-Lorimer quantification using Bruker Esprit software (version 2.0) to obtain the compositional atomic percent (Table S1) based on spectra taken from a DNA triangle and from the adjacent graphene support area (Fig. S8). 
Table S1. Compositional analysis of spectra presented in Fig. S8 expressed as atomic percent.

\begin{tabular}{ccccccccc} 
Spectrum (atomic \%) & $\mathrm{C}$ & $\mathrm{O}$ & $\mathrm{N}$ & $\mathrm{Si}$ & $\mathrm{P}$ & $\mathrm{Cl}$ & $\mathrm{Mg}$ \\
$\begin{array}{c}\text { DNA triangle } \\
\text { Graphene support }\end{array}$ & $\mathbf{7 1 . 6}$ & 15.1 & 7.6 & 0.60 & 0.55 & 0.75 & 3.8 \\
\hline & 86.0 & 8.2 & 2.9 & 1.2 & 0.02 & 0.11 & 1.5
\end{tabular}

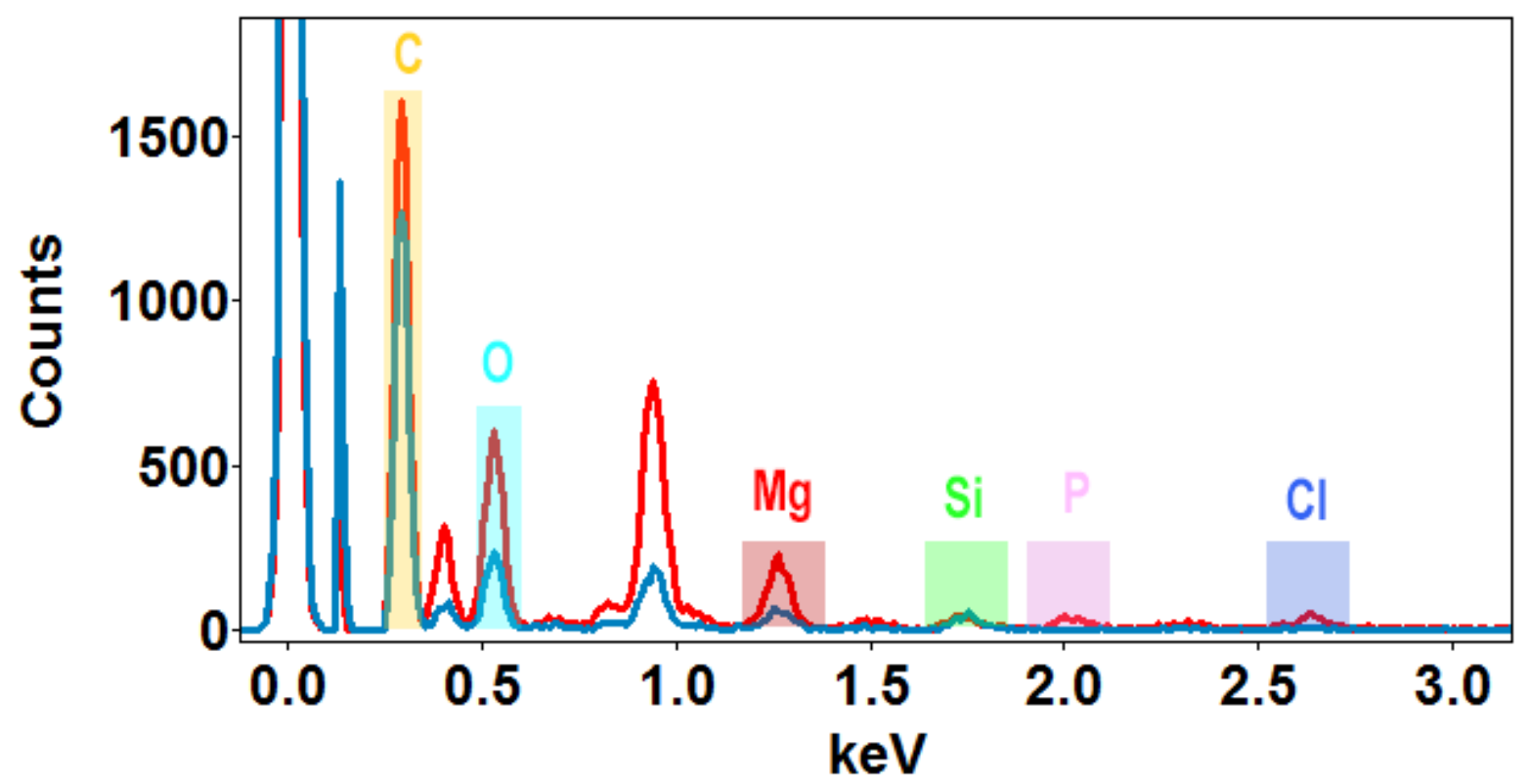

Figure S8. EDS spectra of DNA origami triangle (red) vs. graphene support (blue) with various signature spectroscopic energies shown for different elements.

To test the repeatability of our EDS measurements, we conducted an additional round of experiments using new DNA triangles and freshly-synthesized graphene substrates, but with a more effective buffer exchange process just prior to transfer to the graphene and insertion in the TEM. Representative HAADF and EDS maps for these samples are shown in Fig. S9(A)-(F), and the resulting summed spectra from the DNA-containing and background regions are shown 

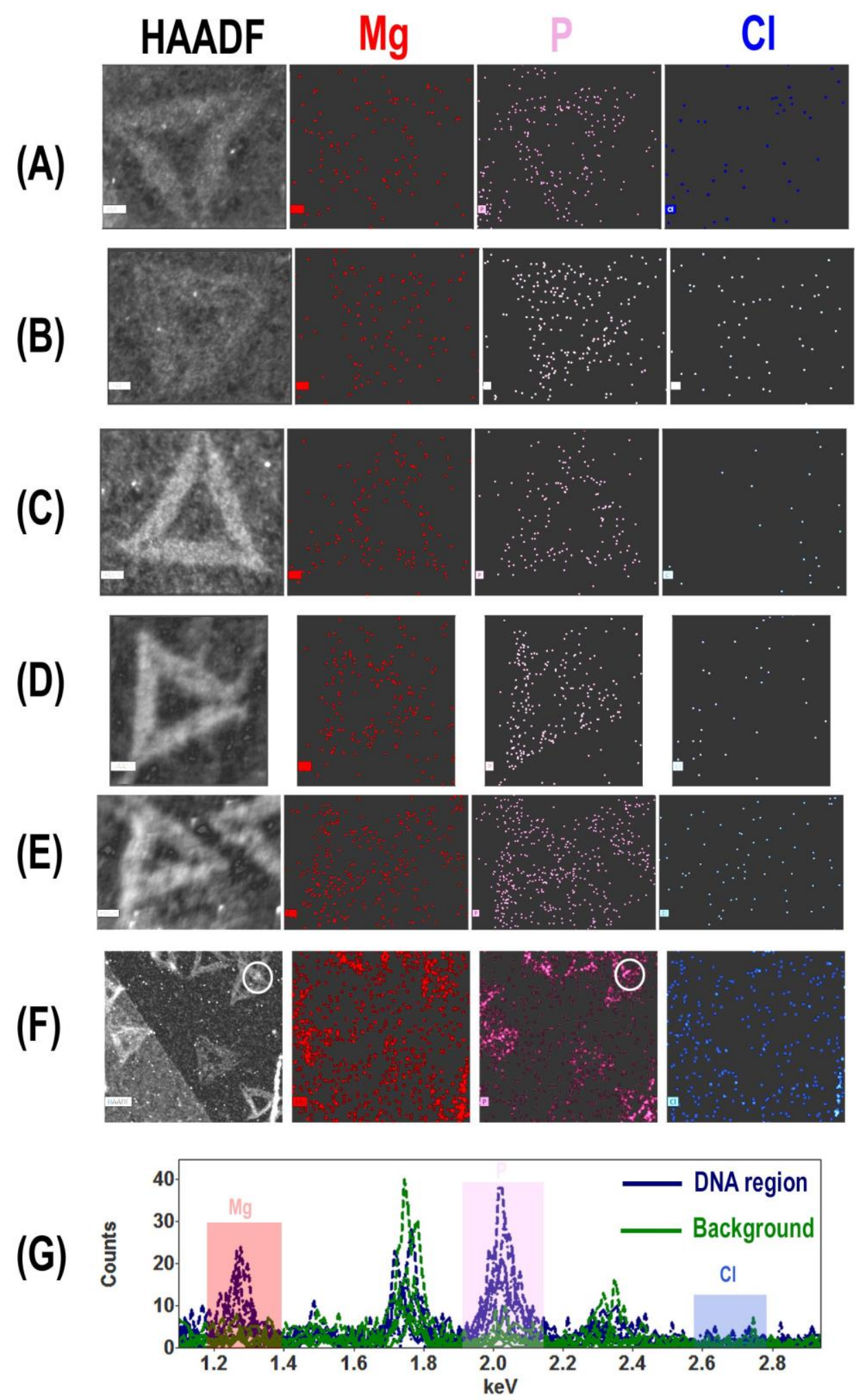

Figure S9. Multiple triangles with $\mathrm{HAADF}, \mathrm{Mg}, \mathrm{P}$, and $\mathrm{Cl}$ with: (A) \& (B) smallest probe, low current; (C) smallest probe, high current; (D) larger probe, high current, (E) 2 triangles; and (F) Multiple triangles. (G) DNA and Background regions summed spectra for all the images shown. 
in Fig. S9(G). As before, clear phosphorus and magnesium signals are seen, however less chlorine is observed which we attribute to the improved buffer exchange. Of note, in the top-right portion of (F), two DNA triangles are actually overlapping, and in comparing the phosphorus content of the 2-layer versus 1-layer triangles, we find that the phosphorus atomic content increases from $0.6 \%$ to $1.0 \%$, which is close to the doubling expected if an additional layer of DNA is present. This is strong support for the correctness of our elemental analysis.

Table S2. Compositional analysis of all EDS maps presented in Fig. S9 expressed as atomic percent.

$\begin{array}{lccccccc}\begin{array}{l}\text { Spectrum } \\ \text { (atomic \%) }\end{array} & \mathbf{C} & \mathbf{O} & \mathbf{N} & \mathbf{S i} & \mathbf{P} & \mathbf{C l} & \mathbf{M g} \\ \begin{array}{l}\text { DNA triangle } \\ \begin{array}{l}\text { Graphene } \\ \text { support }\end{array}\end{array} & 89 \pm 3 & 5.0 \pm 1.3 & 4.2 \pm 0.9 & 0.45 \pm 0.28 & 0.9 \pm 0.7 & 0.07 \pm 0.03 & 0.4 \pm 0.2 \\ & & 2.8 \pm 1.3 & 1.2 \pm 0.5 & 0.6 \pm 0.4 & 0.09 \pm 0.07 & 0.04 \pm 0.03 & 0.12 \pm 0.05\end{array}$

In Table S2, the compositional variation over the triangles in Fig. S9 are shown. The mean atomic percent is shown for each element, with the standard deviation over the 6 different datasets used as a measure of the error. Of course, each atomic percent has its own error, but we note the relatively small errors with the main elements, and the phosphorus signal, while varying, remains well above the graphene support background. Also of note, there is very little to no chlorine present, which differs from the data in Fig. 4 of the manuscript, and which as noted before we attribute to improved buffer exchange. 
Table S3. Total atom counts Fig. S9C following protocol outlined in main text.

$\begin{array}{lccccccc} & \mathbf{C} & \mathbf{O} & \mathbf{N} & \mathbf{S i} & \mathbf{P} & \mathbf{C l} & \mathbf{M g} \\ \begin{array}{l}\text { Triangle region } \\ \text { (ideal) }\end{array} & 289 & 57.7 & 84.6 & 0 & 14.4 & 0 & 0 \\ \begin{array}{l}\text { Triangle region } \\ \text { (EDS) }\end{array} & 847 & 62 & 43 & 9.9 & 14.4 & 0.98 & 6.9 \\ \begin{array}{l}\text { Graphene } \\ \text { support only } \\ \text { (ideal) }\end{array} & 145 & 0 & 0 & 0 & 0 & 0 & 0 \\ \begin{array}{l}\text { Graphene } \\ \text { support only }\end{array} & & & & & & & \\ \text { (EDS) }\end{array}$

In Table S3, the total atom counts for the triangle in Fig. S9C are calculated using the protocol described in the main text (i.e. normalizing to phosphorus atoms and the total number of carbon counts). Here, we note significantly less carbon, oxygen, nitrogen, and chlorine. As mentioned above, we again attribute this to the improved buffer exchange, which leaves both less residual 'contaminants' and less of the solute ions.

In Fig. S10A we show a MAADF image of a DNA origami assembled with barium as the
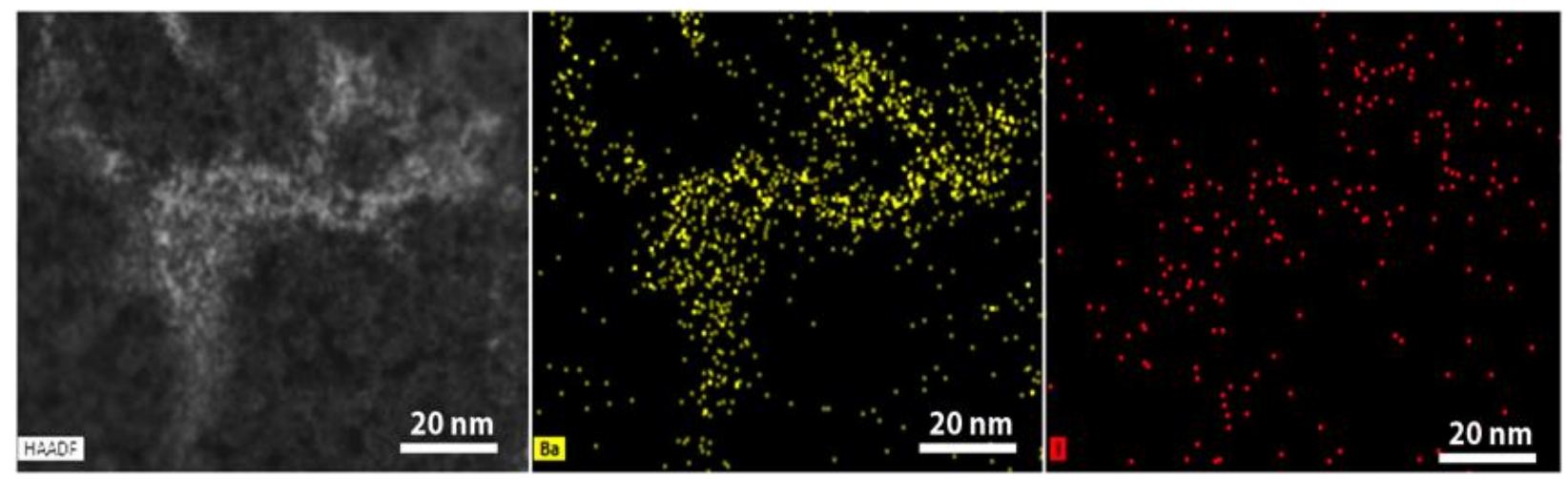

Figure S10. STEM/EDS images of a DNA origami triangle on single-layer graphene: (a) HAADF micrograph, (b) EDS map of barium at L- $\alpha$ energy, and (c) EDS map of iodine L- $\alpha$. 


\section{Supporting Information}

counter-ion as discussed in relation to Fig. S1. The distortion in the DNA structure mentioned earlier is evident here as well. Fig. S10B contains the corresponding EDS map for barium and shows the strong signal resulting from its heavier mass, and like with magnesium (Fig. 4A) shows a clear self-staining effect. Lastly, in Fig. S10C we show the EDS map for iodine, which as noted earlier was added to some of our samples as part of the gel dye. 